\title{
ANÁLISIS POLÍNICO DE MIELES EN LA CABRERA BAJA (MONTES DE LEÓN, ESPAÑA)
}

\author{
Manuel CELIS \& María Josefa DÍEZ
}

RESUMEN. Análisis polínico de mieles en La Cabrera Baja (Montes de León, España).Se ha estudiado el sedimento polínico de siete muestras de miel a microscopía óptica y electrónica de barrido. Las muestras proceden de siete localidades de la comarca de La Cabrera Baja, ubicada en los Montes de León (León, España). Los resultados nos muestran que el néctar de flores es la principal fuente de miel de la zona (EIM/ $P=0.02-0.38$ ).

Identificados 34 tipos polínicos, resultan dos mieles uniflorales de Castaño, una de Mentha y cuatro multiflorales. Los tipos más destacables se corresponden con diversas especies de Mentha, Castanea sativa, Erica australis, E. arborea, Campanula spp., Reseda spp., Rubus spp., y Salix spp. como recursos nectaríferos para Apis mellifera. Casi todas estas especies, pero fundamentalmente diversos taxones de la tribu Genisteae, son las fuentes de polen en la comarca.

Palabras clave. Melitopalinología, miel, León, España, apicultura, polen, palinología, Apis mellifera.

SUMMARY. Pollen analysis of honeys from La Cabrera Baja (Montes de León, Spain). Seven honey sample have been studied by light and scanning electron microscopy, from seven localities of the Montes de León region (Leon, Spain). The results show that the nectar from flowers is the main source in the region $(\mathrm{HDE} / \mathrm{P}=0.02-0.38)$

Thirty four pollen types have been identified which show two monofloral (from Chestnut and one from Mint) and four multifloral honey. The main types are different species of Mentha, Castanea sativa, Erica australis, E. arborea, Campanula spp., Reseda spp., Rubus spp., and Salix spp. like the most important plants supplying nectar for Apis mellifera. All these taxa, but mainly different species of tribu Genisteae, are the principal pollen sources in the region.

Key words. Melisopalynology, honey, León, Spain, beekeeping, pollen, palynology, Apis mellifera.

\section{INTRODUCCIÓN}

La comarca de La Cabrera, al SW de León, es una de las más montañosas de la provincia. Se encuentra en la transición de las regiones florísticas Mediterránea y Eurosiberiana.

En la actualidad, los suelos dominantes son marcadamente pobres en nutrientes, con perfiles raramente superiores a los $25 \mathrm{~cm}$.

Con una altura media por encima de los $1000 \mathrm{~m}$, y un paisaje lleno de valles de fondo seco, escobios y fuertes pendientes, la explotación agraria está forzosamente limitada a no más del $10 \%$ del terreno geográfico, prácticamente el más próximo a las poblaciones 
(Cabero, 1.980).

La vegetación está compuesta por robles (Quercus robur, Quercus pyrenaica), castaños (Castanea sativa), antes muy extendidos; encinas (Quercus ilex) y alcornoques ( $Q$. suber), como reminiscencias claramente mediterráneas, y especies atlánticas como los nogales (Juglans regia), tejos (Taxus baccata), acebos (Ilex aquifolium) y abedules (Betula celtiberica).

Sin embargo, la primera componente paisajística es el matorral, de origen antropógeno y fundamentalmente constituido por «urces» o brezos (Erica spp.), escobas (Cytisus scoparius) y otras genisteas como Adenocarpus complicatus, Genista hystrix, $G$. sanabrensis, G. tridentata, etc.

El tipo de agricultura que se practica es el policultivo de autoabastecimiento o de subsistencia, que ofrece un paisaje agrícola fragmentado y muy diverso en especies cultivadas.

Pensamos, por todo ello, que la apicultura es una buena alternativa y/o complemento de uso de los recursos naturales de este territorio. De ahí el interés que tiene conocer la flora de interés apícola de la comarca.

Desde 1950 en que Vieitez realizó el primer estudio del sedimento polínico de mieles españolas, diversos autores han realizado trabajos similares. Destacamos los de Sánchez Sanchez (1.982), Sánchez \& Saenz (1982), Aira et al. (1990), Janeiro et al. (1992) y Valencia Barrera (1992), que analizaron mieles procedentes de Galicia y León, en zonas más o menos próximas a la de este estudio.

\section{MATERIAL Y MÉTODOS}

El material estudiado procede de siete muestras de miel correspondiente a la cosecha de 1.990 para autoconsumo de los apicultores de las siguientes localidades: Ambasaguas (AMB1 y AMB2), La Baña (LB), Llamas
(LLA), Marrubio (MAR), Robledo de Losada (RL) y Vega de Yeras (VY).

El análisis cuantitativo de las muestras se ha efectuado en preparaciones microscópicas procedentes del sedimento obtenido del lavado y centrifugado de la miel. El análisis cualitativo se ha llevado a cabo sobre preparaciones acetolizadas (Erdtman, 1960). Siguiendo las indicaciones de Vergeron (1964) y Louveauxe et al. (1978) se han contado e identificado al menos 1200 granos de polen repartidos en cuatro preparaciones de cada muestra.

Para la identificación del polen se ha usado principalmente el «Atlas Polínico de Andalucía Occidental» (Valdés et al., 1987) y las preparaciones de referencia de la palinoteca del Departamento de Biología Vegetal de la Universidad de Sevilla, así como de algunas preparaciones acetolizadas de polen de plantas procedentes de las zonas circundantes a las colmenas. Todas las muestras se han observado además al microscopio electrónico de barrido (MEB) para confirmar y/o determinar los tipos polínicos identificados con el microscopio óptico (MO).

\section{RESULTADOS}

Los resultados del análisis cuantitativo, junto a la relación entre los elementos indicadores de mielada y el polen de plantas nectaríferas (EIM/PN) se presentan en la tabla 1.

El sedimento de todas las muestras está formado por granos de polen de plantas poliníferas y nectaríferas, así como por elementos indicadores de mielada, concretamente hifas y esporas de hongos.

El número de granos de polen en $10 \mathrm{gr}$ de miel (NGP) oscila desde los 13.200 de la muestra AMB2 hasta los 2.313.000 en LB. Igualmente, existe una amplia variabilidad en el número de elementos indicadores de mielada: 2.200 en AMB2 y 60.000 en MA, diferencias 


\begin{tabular}{lccccc}
\hline M./M. & N.G.P. & N.E.I.M. & N.E.B.T. & EIM/PN. & CLASE \\
\hline AMB1 & 21.200 & 4.200 & 25.400 & 0,38 & II \\
AMB2 & 13.200 & 2.200 & 15.400 & 0,19 & I \\
LB & 2.313 .000 & 45.000 & 2.358 .000 & - & V \\
LLA & 116.000 & 4.200 & 120.200 & 0,07 & III \\
MAR & 1.470 .000 & 60.000 & 1.530 .000 & - & V \\
ROB & 108.600 & 7.800 & 116.400 & 0,13 & III \\
VY & 222.000 & 25.000 & 247.000 & 0,12 & III \\
\hline
\end{tabular}

Tabla 1. Resultados del análisis cuantitativo. MM: muestra de miel; NGP: número de granos de polen; NEIM: número de elementos indicadores de mielada; NEBT: número de elementos botánicos; EIM: elementos indicadores de mielada; PN: número de granos de polen de plantas nectaríferas. Results of cuantitative analysis. MM: honey sample; NGP: Number of pollen grain; NEIM: Number of honeydeew elements; NEBT: Number of botanical constituents; EIM: Honeydeew elements; PN: Number of pollen grains from nectariferous plants.

\begin{tabular}{|c|c|c|c|c|c|c|c|}
\hline TIPO POLINICO/MUESTRA & AMB1 & AMB2 & LB & LLA & MAR & RL & VY \\
\hline T. Apiaceae & 4 & 1,5 & + & + & + & - & + \\
\hline T. Artemisia campestris & + & - & - & - & 3 & - & - \\
\hline T. Calendula arvensis & + & - & - & 1 & 1 & - & - \\
\hline T. Centaurea & + & - & - & - & + & - & - \\
\hline T. Echium vulgare & - & 2 & - & - & - & + & - \\
\hline T. Campanula erinus & 1 & 11,5 & - & 1 & 5 & + & 5 \\
\hline T. Corrigiola telephiifolia & 6,5 & - & - & - & - & - & - \\
\hline T. Chenopodium I & 1,5 & - & - & - & 1 & + & - \\
\hline T. Chenopodium II & - & - & - & - & + & - & - \\
\hline T. Cistus ladanifer & 5,5 & - & + & - & - & - & 1,5 \\
\hline T. Cistus psilosepalus & 5,5 & - & - & - & - & - & + \\
\hline T. Halimium halimifolium & - & - & + & 4,5 & + & - & - \\
\hline T. Capsella bursa-pastoris & - & - & - & - & + & 1 & + \\
\hline T. Erica arborea & 2,5 & 10 & + & 9,5 & - & 2,5 & 1,5 \\
\hline T. Erica australis & 4,5 & 20,5 & + & 8 & 1 & 5 & 1,5 \\
\hline T. Cytisus scoparius I & 31 & 4 & 4 & 33 & 7,5 & 19 & 3 \\
\hline T. Cytisus scoparius II & 2 & 1,5 & - & 5 & - & 14,5 & + \\
\hline T. Lotus creticus & - & - & - & 6,5 & - & - & - \\
\hline T. Castanea sativa & 10 & 6,5 & 94 & + & 34,5 & 11 & 83,5 \\
\hline T. Hypericum & - & 3 & - & - & - & - & - \\
\hline T. Lavandula stoechas & - & - & - & - & + & + & - \\
\hline T. Mentha I & 3,5 & 8 & + & - & + & 14 & - \\
\hline T. Mentha II & 2,5 & 6 & - & - & + & 2,5 & - \\
\hline T. Mentha III & 1,5 & - & - & 1 & + & - & - \\
\hline T. Mentha IV & 1,5 & - & - & + & 2 & - & - \\
\hline T. Sideritis & - & 4 & - & - & - & - & - \\
\hline T. Fraxinus & - & - & - & 1 & + & 6,5 & - \\
\hline T. Chelidonium majus & - & - & - & 1 & - & - & 1,5 \\
\hline T. Papaver & - & + & - & + & + & 1 & - \\
\hline T. Plantago & 3 & - & - & + & 6,5 & + & - \\
\hline T. Reseda luteola & 2,5 & 16,5 & - & 29 & + & - & + \\
\hline T. Rubus ulmifolius & - & - & - & - & 28 & 17 & - \\
\hline T. Salix fragilis & 10 & + & - & + & + & + & - \\
\hline T. Verbascum pulverulentum & - & - & - & - & 3 & - & - \\
\hline
\end{tabular}

Tabla 2. Resultados del análisis cualitativo en \%. (+): presencia del tipo aludido, con porcentaje inferior al 1\%. (-): ausencia del tipo aludido. Results of cualitative analysis in \%; $((+)$ presence of the pollen type, with percentage lower than 1\%; -): absence of the pollen type. 


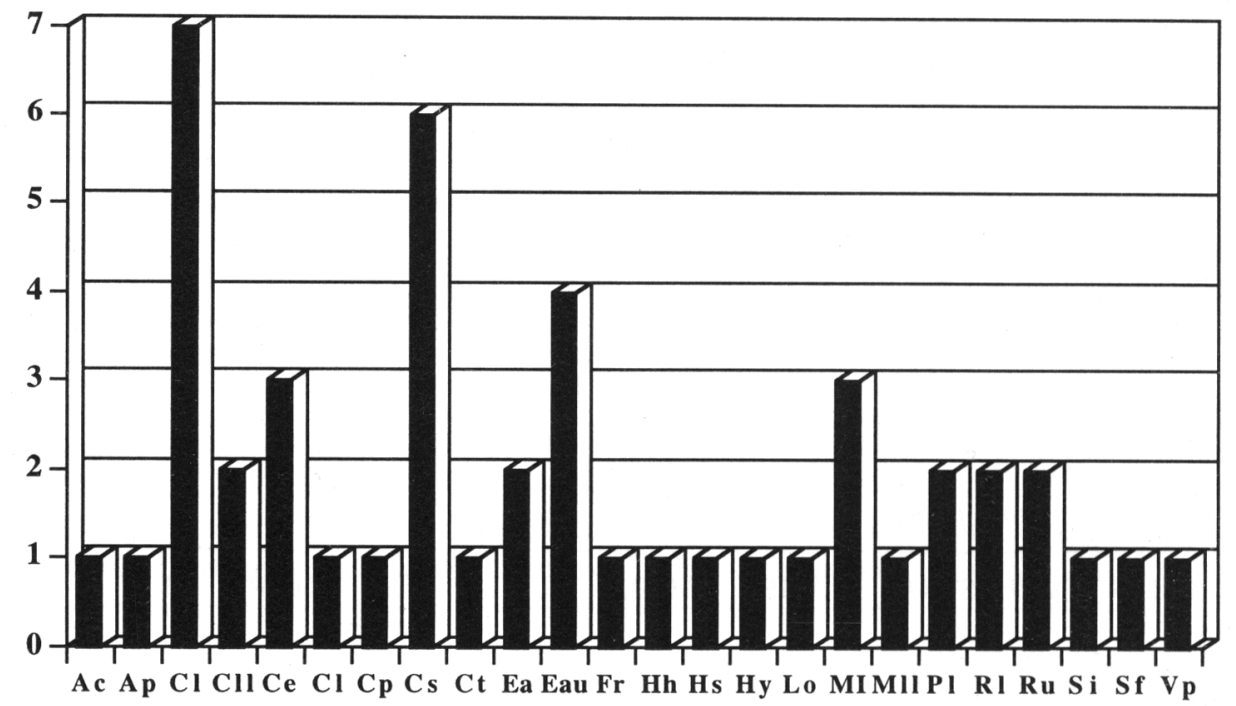

Figura 1. Número de muestras en que aparece cada tipo polínico con un porcentaje superior al $3 \%$. Ac, Artemisia campestris; Ap, Apiaceae; Ce, Campanula erinus; CI, Cytisus I; CII, Cytisus II; Cl, Cistus ladanifer; $\mathrm{Cp}$, Cistus psilosepalus; $\mathrm{Cs}$, Castanea sativa; $\mathrm{Ct}$, Corrigiola telphiifolia; Ear, Erica arborea; Eau, Erica austrlis; Fr, Fraxinus; Hh, Halimium halimifolium; Hs, Helianthemum siriacum; Hy, Hypericum; Lo, Lotus; MI, Mentha I; MII, Mentha II; Pl, Plantago; RI, Reseda luteola; Ru, Rubus ulmifolius; Si, Sideritis; Sf, Salix fragilis; Vp, Verbascum pulverulentum. Number of samples with every pollen type represented above $3 \%$.

que podrían deberse a variaciones locales, ya que todas las muestras fueron extraidas en Septiembre y las alzas colocadas en Mayo.

Las mieles presentan una amplia variedad de sedimento polínico; clases I, II, III y V, si bien en la mayoría es abundante: tres pertenecen a la clase III y dos a la V (Maurizio, 1.949, sec. Maurizio, 1.979).

Los resultados del análisis cualitativo se presentan en el tabla 2. Se han identificado 34 tipos polínicos, considerando como mínimo un porcentaje de aparición del $1 \%$ en cada muestra. Se expresa con un signo «+» la presencia del tipo aludido, con porcentaje inferior al $1 \%$.

La figura 1 representa el número de muestras en que aparece cada tipo polínico. Se han representado sólo aquellos tipos que tienen una frecuencia superior al $3 \%$.

\section{DISCUSIÓN}

Por los resultados obtenidos todas las mieles han resultado ser de néctar (EIM/PN < 1), si bien el considerable número de EIM presentes en las muestras de LB y MA (45.000 y 60.000 , respectivamente) hace pensar que la mielada como fuente nectarífera de la zona podría tener cierta importacia local.

Las muestras de LB y VY pueden denominarse, según sus espectros polínicos, como mieles monoflorales de Castaño (Crane, 1979), presente en un $94 \%$ y $83,5 \%$ respectivamente. La miel de $\mathrm{LB}$, de Clase V ha sido extraida por prensado, mientras que la de VY (clase III) lo ha sido por centrifugado.

Las dos muestras de Ambasaguas (AMB1 y AMB2) son las que presentan menos 
sedimento polínico. Pertenecen a las clases I y II, ambas obtenidas por centrifugado (Crane, 1979). Las dos han resultado ser multiflorales o milflores, pero a pesar de la proximidad relativa de las colmenas, los espectros polínicos han resultado ser diferentes. En AMB 1, el polen de Cytisus (I y II) representa el 33\% del total, considerándose a esta planta como la principal fuente de polen en la colmena, junto a Cistus (C. ladanifer y $C$. psilosepalus) que representa el $11 \%$. En cambio, en AMB2, Cytisus representa el $5.5 \%$ y las Cistáceas, presentes en un $3 \%$, corresponden a Helianthemum syriacum. No obstante, en ambas muestras las Labiadas han resultado ser importantes fuentes de néctar, representadas por un $9 \%$ (tipos Mentha I,II,III,y IV) en AMB1 y un $18 \%$ (Mentha I y II y Sideritis) en AMB2 (no olvidemos el carácter de infrarepresentación del polen de estas especies) (Crane, 1979). La miel AMB2 podría considerarse como monofloral de Mentha.

La muestra LLA ha resultado ser de la clase III, pero el propio apicultor manifestó que esta procedía de una mezcla de miel prensada y centrifugada. Esta miel multifloral se caracteriza por la presencia de Cytisus (I y II) en un $38 \%$ y Reseda en un $29 \%$, que son las fuentes de polen más importantes. Las especies que han aportado néctar a la miel son diversas. Todas aparecen en porcentajes relativamente bajos: Erica australis $(9,5 \%)$, Erica arborea (8\%), Campanula sp. (1\%) y Mentha III (1\%), lo cual parece coherente con la procedencia mixta de centrifugado y prensado de esta miel (Crane, 1979).

La muestra MA, de la clase V y por tanto obtenida por prensado, también ha resultado ser multifloral. La fuente de néctar más importante es Rubus ulmifolius (28\%), pero han contribuido también Castanea sativa $(34,5 \%)$, especie claramente hiperrepresentada, Campanula spp. (5\%) y Mentha IV (2\%). Rubus ulmifolius y Castanea sativa destacan también como fuentes importantes de polen.
Por último, la muestra de RL, de la clase III y, por tanto, obtenida por centrifugado, se ha considerado de Mentha o mixta de Mentha y Rubus, ya que sus tipos polínicos son los más abundantes ( $17 \%$ y $16,5 \%$ respectivamente) en cuanto a especies nectaríferas se refiere. Como fuente principal de polen encontramos Cytisus I y II con un $33,5 \%$.

A la vista de estos resultados, se concluye que las principales fuentes de néctar encontradas son Castanea sativa y diversas labiadas, fundamentalmente especies de Mentha, que producen mieles monoflorales, Erica australis, Erica arborea y Rubus ulmifolius. Otros tipos nectaríferos son Campanula spp, Salix spp y Reseda luteola.

Sin duda, la fuente de polen por excelencia corresponde al tipo Cytisus, fundamentalmente Cytisus scoparius, muy abundante en la zona; además, tienen importancia Castanea sativa, Reseda (probablemente Reseda luteola), especies de Erica (E. arborea y E. australis) y, en menor medida, Campanula spp., Salix spp., Plantago spp., algunas Cistaceas (Cistus ladanifer, C. psilosepalus, Halimium Halimifolium), etc.

Nos ha extrañado la ausencia de polen de plantas de cultivo (especies de Phaseolus, Solanum, Capsicum) dada su proximidad a las colmenas, su favorable fenología floral y su interés apícola (Guillén, 1990; Rabinowitck et al., 1993).

Estos resultados nos conducen a pensar que la potencialidad apícola de la comarca no está siendo aprovechada adecuadamente. Las colmenas están ubicadas en las proximidades de los núcleos de población, por lo que el abundante matorral de brezo, que invade todo el monte, es infrautilizado por las abejas. Recordamos que a pesar de que el polen de Erica está presente en todas las muestras, no se ha obtenido ninguna miel monofloral de brezo, lo que podría conseguirse con facilidad colocando las colmenas en monte abierto.

Igualmente, a pesar de los pocos castaños 
que van quedando, debido sobre todo al abandono, y dado que se trata de una especie sumamente visitada por Apis mellifera, pueden conseguirse mieles monoflorales de esta especie sin más que situar las colmenas en su proximidad y en la época apropiada. No obstante, hay que mencionar que en general, esta miel es poco apreciada (Ernos, 1980).

Por otro lado, la colocación de las alzas en la zona se realiza generalmente en Mayo, y teniendo en cuenta las condiciones climáticas de la misma (comentadas en la introducción) y la presencia de especies de Erica, frutales del género Prunus como el cerezo y herbáceas de cultivo como Brassica napus, de floración temprana, podrían colocarse antes, elevando así la producción.

Por último, pensamos que en la zona pueden obtenerse fácilmente mieles monoflorales (o al menos mixtas) de Mentha. Las especies de este género, especialmente Mentha suaveolens y M. pulegium, así como diversas especies de Thymus son muy frecuentes, y hemos observado que en las colmenas próximas a poblaciones de estas especies, AMB1, AMB2 y RL, los porcentajes encontrados son considerables.

AGRADECIMIENTOS. Los autores desean expresar su agradecimiento a todos los apicultores que facilitaron desinteresadamente sus muestras de miel.

\section{BIBLIOGRAFÍA}

AIRA, M.J., P. RAMIL-REGO y M.P. SAA-OTERO -1990- Identificación polínica de Ericaceas en mieles gallegas. Acta Bot. Malacitana, 15: 27-32

CABERO, V. -1980- Espacio agrario y economía de subsistencia en las montañas GaláicoLeonesas: La Cabrera. Universidad de Salamanca. Serie Varia. Temas científicos y literarios, 29. Institución «Fray Bernardino de Sahagún» C.S.I.C.- León.

CRANE, E. -1979- Honey. A comprehensive Survey. Heinemann. pp. 256-257. London.
ERDTMAN, G. -1960- The acetolysis method. A revised description. Svensk. Bot. Tidskr., 54(4): 561-564.

ERNOS, V. -1.980- Hay dinero y salud en la abeja. Ed. Sintes. pp. 40-41. Hospitalet.

GUILLÉN, A. -1990- Estudio de la flora de interés apícola de la provincia de Zamora. Diputación de Zamora. Zamora.

JANEIRO, B., J. AMIGO y M.J. AIRA -1992Estudio polínico secuencial de la miel de Paradapiñol (Quiroga, Lugo, España) y su relación con la flora melífera de la zona. Bol. $R$. Soc.Esp. Hist. Nat. (Sect. Biol.) 88(1-4): 213-221.

LOUVEAUX, J.A., A. MAURIZIO \& G. VORWOHL -1978-Methods of melissopalynology. Bee World, 59: 139-157.

MAURIZIO, A. -1979- Microscopy of honey. In E. CRANE (ed.) Honey. A comprehensive survey. Heinemann. London. 240-257.

RABINOWITCH, H.D., A. FAHN, T. MEIR \& Y. LENSKY -1993-Flower and nectar attributes of pepper (Capsicum annuum L.) plants in relation to their attractiveness to honeybees (Apis mellifera L.) Ann. Appl. Biol., 123: 221-232.

SÁNCHEZ, C. y C. SAENZ -1982- Análisis polínico de mieles de Pontevedra (España). Lazaroa, 4: 253-268.

SÁNCHEZ SÁNCHEZ, J. -1982- Análisis polínico de mieles de la provincia de Salamanca. Universidad de Salamanca. Salamanca.

VALDÉS, B., M.J. DÍEZ \& I. FERNÁNDEZ - 1987 Atlas polínico de Andalucía Occidental. Inst. Des. Regional y Diputación de Cádiz. Sevilla.

VALENCIA-BARRERA, R.M. -1992- Estudio palinológico de mieles de la provincia de León. Tesis doctoral, microficha $\mathrm{n}^{\circ}$ 93. Universidad de León. León.

VERGERON, Ph. -1964- Interpretation statistique des résultats en matiére d'analyse pollinique des miels. Ann. Abeille, 7(4): 349-364.

VIEITEZ, E. -1950- Palynological observations on some Spanish honeys. Bull. Torrey Bot. Club, 77(6): 495-502.

Aceptado para su publicación en Noviembre de 1994

Dirección de los autores. Depto. de Biología Vegetal y Ecología (Botánica). Universidad de Sevilla. Apdo. 1095. 41080, Sevilla 